\title{
Coexistence of tinnitus and hyperacusis in individuals with auditory dys-synchrony: A single case study
}

\author{
K. N. Megha, Sugathan Adithya, K. P. Keerthana, Sreeraj Konadath* \\ All India Institute of Speech and Hearing, Manasagangothri, Mysuru, India
}

\begin{abstract}
Summary Certain clinical pathologies affecting the ear and hearing mechanism may co-exist. It is necessary to probe in detail into such conditions so that the pathophysiology is well understood. This research paper through a single case study tries to explain the probable pathophysiology behind coexistence of three different clinical conditions namely auditory dys-synchrony, hyperacusis and tinnitus. These conditions are common in the clinics, but the coexistence of all the three is rare and demands explanation beyond what is available in the literature. The assumed model highlights involvement of the outer hair cell's motor function in the cochlea along with the auditory central gain mechanism to explain possible pathophysiology behind coexistence of the three conditions. This model will provide insight into the probable link between the contribution of peripheral and central structures of hearing in generating tinnitus and hyperacusis in individuals having auditory dys-synchrony.
\end{abstract}

Keywords: Auditory dys-synchrony, tinnitus, hyperacusis

\section{Introduction}

Communication and exchange of information is an important part of our everyday lives. Having a hearing loss can significantly impact a person's ability to communicate with others, leading to a reduced quality of life. People with hearing loss face difficulties in their emotional, social and physical well-being (1). There are different types of hearing loss namely conductive, sensorineural and mixed hearing loss, each of which has different effects on living. Sensorineural hearing loss happens due to mutilation in the cochlea, that is the inner ear, or to the nerve pathways from the inner ear to the brain (2). Auditory dys-synchrony/neuropathy (AN/AD) is a hearing impairment where outer hair cell amplification is normal, but auditory pathway's afferent neural conduction is disordered (3). The diagnosis is based on the integrity revealed by the presence of otoacoustic emission by the outer hair cell, absence or severe abnormality of the auditory brainstem responses, impaired speech perception and acoustic middle ear

Released online in J-STAGE as advance publication January 27, 2016.

*Address correspondence to:

Mr. Sreeraj Konadath, All India Institute of Speech and Hearing, Manasagangothri, Mysuru 570 006, India.

E-mail: sreerajkonadath@aiishmysore.in reflexes showing absence of responses or marked elevation (4). 1 in 200 hearing impaired children had an audiological indication of dys-synchrony (5). In individuals with sensorineural hearing loss in Mysore, the prevalence was around 1 in 183 (6). Most of the individuals with auditory dys-synchrony reported difficulty in understanding speech, and tinnitus was found to be one of the complaints $(6,7)$. It was reported that $50 \%$ of individuals with auditory dys-synchrony had bilateral tinnitus (O).

Tinnitus is described as a conscious expression of a sound which instigates in an involuntary manner in the head of its owner, or might appear to him to do so (8). It is due to over activity of the cochlear hair cells that leads to growth in the (spontaneous) firing rate of primary auditory nerve fibers. The generators of tinnitus are ideally located in the auditory pathway, and are explained using various theories like edge theory and discordant damage theory. Those present in the central auditory system are explained in terms of other studies like, the auditory pathway theory, the crosstalk theory, the limbic and autonomic nervous system, and the somatosensory system (9). Prevalence of tinnitus ranged between 4.4 and $15.1 \%$ in adults (10). Tinnitus can have a significant impact on our life, although most have learned to live with it. It can disturb concentration and cause sleeping problems, depression and stress. The incidence of hyperacusis is very high among individuals 
with tinnitus; 40 and $86 \%$ of those who have tinnitus also have hyperacusis (11).

Hyperacusis is defined as an abnormal lowered tolerance to sound (12). Hyperacusis is an abnormally strong reaction occurring within the auditory pathways resulting from exposure to moderate sound; as a consequence, patients express reduced tolerance to supra-threshold sounds (13). As a result of compensatory increase in neural amplification in the auditory system which tries to compensate for a loss of sensory input from the cochlea tinnitus and hyperacusis might result (14). Lesions causing tinnitus could have some direct effect on the loudness perception that leads to a reduced uncomfortable loudness level (15).

The co-existence of auditory dys-synchrony, tinnitus and hyperacusis is very rare. This article provides information on the co-existence of all three conditions with the help of a case study outcome of an adult who was diagnosed as auditory dys-synchrony with tinnitus and hyperacusis. Also, it explains a hypothetical model to describe their co-existence.

\section{Case report}

\subsection{Assessment}

Evidence from the literature for the co-existence of all three conditions has not been reported. Because all three conditions are reported to All India Institute of Speech and Hearing (AIISH), it was necessary to find the reason for the occurrence of these conditions in the same person. The patient is a 24 year-old male who was previously diagnosed as having mild to moderately severe sensorineural hearing loss in both ears. The patient underwent different medications for the problems listed, even though no other non-medical treatments were taken.

The patient complained of reduced hearing sensitivity in both ears, difficulty understanding speech, severe tinnitus (continuous ringing type) and intolerance to sounds. However, the patient did not mention intolerance to specific sounds. The patient also had severe psychological issues due to continuous ringing tinnitus and also had sleep disturbances due to the same problem. He exhibited all these signs since 5 years old and had consulted many centers but failed to get a solution. Computed Tomography (CT) scan of the brain done at Bengaluru, India indicated calcified granuloma in left parietal lobe $(5.6 \times 4.1 \mathrm{~cm})$ with no peri-lesional edema. The EEG results indicated no electrographic evidence of focal structural abnormality. Diagnosis from audiological evaluations done at different centers is given in Table 1.

Late Latency Potential (LLR) was done to find whether the patient is a candidate for cochlear implant or not because he was not benefiting from a hearing aid (combination device). The patient had undergone
Table 1. Summary of results of audiological evaluations done at different centers

\begin{tabular}{ll}
\hline Date (Center) & Provisional diagnosis \\
\hline $\begin{array}{l}\text { 12/04/2011 } \\
(\text { Centre 1) }\end{array}$ & $\begin{array}{l}\text { Right ear: Mild SNHL } \\
\text { Left ear: Moderate SNHL (raising pattern) }\end{array}$ \\
$02 / 06 / 2011$ & $\begin{array}{l}\text { Right ear: Moderate SNHL } \\
\text { (Centre 2) }\end{array}$ \\
$\begin{array}{l}\text { Left ear: Moderately severe SNHL } \\
\text { (Centre 3) }\end{array}$ & $\begin{array}{l}\text { Right ear: Moderate SNHL } \\
\text { Left ear: Moderately severe SNHL } \\
\text { Bilateral retro-cochlear pathology }\end{array}$ \\
& Right ear: Moderate SNHL \\
$($ Centre 4$)$ & Left ear: Moderate- Moderately severe SNHL \\
\hline
\end{tabular}

a hearing aid trial on January 9, 2015. Different combination devices were tested which included two programs. First program wherein only amplification was provided and the second program where only noise was given without amplification.

\subsection{Diagnostic tests and interpretations}

The diagnostic tests used in the present patient included pure-tone audiometry, auditory brainstem responses, otoacoustic emissions and middle ear reflexes. The patient underwent pure-tone audiometry on January 8, 2015 at AIISH. The diagnosis made was mild hearing loss in right ear and moderate sensorineural hearing loss in left ear (rising pattern audiogram) with auditory dyssynchrony and hyperacusis. Speech identification scores were poor and worsened in a speech in noise test (SPIN). The middle ear reflexes were absent with the presence of robust otoacoustic emissions. Auditory brainstem response $(\mathrm{ABR})$ - site of lesion was performed and the results were absence of $\mathrm{ABR}$ for click stimulus at both 11.1 and 90.1 repetition rate. The results obtained in the evaluations done at AIISH are provided in Table 2.

There was absence of middle ear reflexes, otoacoustic emissions and ABR in this patient. This might be because of very few inner and outer hair cells remaining. If only outer hair cells remain, emissions are seen but without synchronous firing of the neural elements stemming from inner hair cells, which is the hallmark of AN/AD (16). As the patient complained of tinnitus, tinnitus evaluation was done and there was absence of residual inhibition in both ears. Tinnitus evaluation was done and frequency and pitch matching was done at 2,000 $\mathrm{Hz}$ at $80 \mathrm{dBHL}$ and $750 \mathrm{~Hz}$ at 70 $\mathrm{dBHL}$ in right and left ear respectively. Uncomfortable level of loudness for speech stimulus was found to be $>90$. However, the patient had a tolerance problem at higher frequencies when administered with Johnson's Hyperacusis test. The loudness discomfort levels were obtained and the Johnson's Hyperacusis Quotient (JHQ) was 50-74 which indicated moderate hyperacusis in both ears. The patient obtained a score of 84 when 
Table 2. Summary of results of audiological evaluations done at AIISH

\begin{tabular}{lll}
\hline Date & Audiological evaluations & Results \\
\hline $08 / 01 / 2015$ & Pure-tone audiometry & $\begin{array}{l}\text { Right ear: Mild hearing loss } \\
\text { Left ear: Moderate sensorineural hearing loss }\end{array}$ \\
& Speech audiometry & Poor speech identification scores \\
& Immittance & Absence of reflexes in both ears \\
& Otoacoustic emissions & Presence of robust OAE's in both ears \\
& Auditory Brainstem Response (SOL) & Absence of ABR in both ears \\
& Tinnitus evaluation (Residual inhibition) & Absence of residual inhibition \\
$09 / 01 / 2015$ & Tinnitus Handicap Inventory & $\begin{array}{l}\text { Score:- 84 } \\
\text { Impression: Catastrophic tinnitus }\end{array}$ \\
& Johnson's Hyperacusis Test & JHQ:- 50-74 \\
& Pure-tone audiometry & Moderate sensorineural hearing loss in both ears \\
& Speech audiometry & Poor SIS scores \\
& Immittance & Absence of reflexes in both ears \\
& Otoacoustic emissions & Presence of robust OAE's \\
LLR & Presence of LLR in both ears \\
\hline
\end{tabular}

administered the Tinnitus Handicap Inventory (17) and the impression made was catastrophic tinnitus.

The management option initially tried was a combination device. A combination device is a hearing aid with a built-in sound/noise generator for treating tinnitus. However, the performance using this device was not satisfactory as the patient also had auditory dyssynchrony. The patient was re-evaluated after 3 months and the pure-tone thresholds were slightly elevated. Both right and left ear had moderate sensorineural hearing loss. As the patient was not benefited by the hearing aids, LLR was done to check whether patient is a good candidate for cochlear implantation. The LLR was present in both ears for tone burst and also for speech stimulus.

\section{Discussion}

The results of the present study show that the patient had all the aforementioned clinical conditions coexisting. The possible reason for the co-existence is explained based on the pathophysiology of these conditions through a hypothetical model generated for the same (Figure 1). Starr et al.(4) in 1996 defined "auditory dys-synchrony as a condition to describe hearing loss characterized by normal or near normal hair cell function and absent or abnormal auditory function". The etiology can be at the junction between the cochlear branch of VIII nerve and the inner hair cell that is the synapse, the inner hair cell or at the VIII nerve itself $(4,18)((\mathrm{A})$ in Figure 1).

Auditory dys-synchrony is characterized by absent or abnormal ABR, presence of cochlear microphonics
(CM), and otoacoustic emissions (OAEs) (4,19-21). A normal middle ear function is reflected by normal outer hair cell function in the cochlea inferred through oto-acoustic emissions. But, in some patients who have normal otoacoustic emissions, there will be either absence of inner hair cells or compromised neural synchrony $(4,18)$. In the organ of corti the outer hair cell's motile function is involved in both increasing the activity on the basilar membrane accompanying low intensity sound and with the fine tuning of the activity (22). The efferent fibers innervate the $\mathrm{OHC}$ from the central auditory system in large part, which will analyze the sound environment and will influence the cochlear function accordingly. The stereocilia and cuticular plate region has an active bidirectional transduction mechanism with the OHC. First, the mechanical energy applied to the hair bundle is transduced into electrochemical energy of receptor potential/current of the $\mathrm{OHC}$ to drive the hair bundle mechanically. The active mechanical force at the $\mathrm{OHC}$ hair bundle would push against the tectorial membrane and drive the organ of corti, conceivably reducing the mechanical damping associated with vibration of the organ of corti. This property of $\mathrm{OHC}$ gives rise to active/non-linear biomechanical behavior of the cochlea, and is highly vulnerable physiologically (23).

The mechanical response of the cochlea to the receptoneural signals by the IHC subsystem is to transmit the auditory information with high spatial and temporal resolution via the bulk of the afferent cochlear nerve channel to the higher centers of the brain. It has been determined that stereocilia of the outer hair cell are firmly rooted in the overlying tectorial membrane 


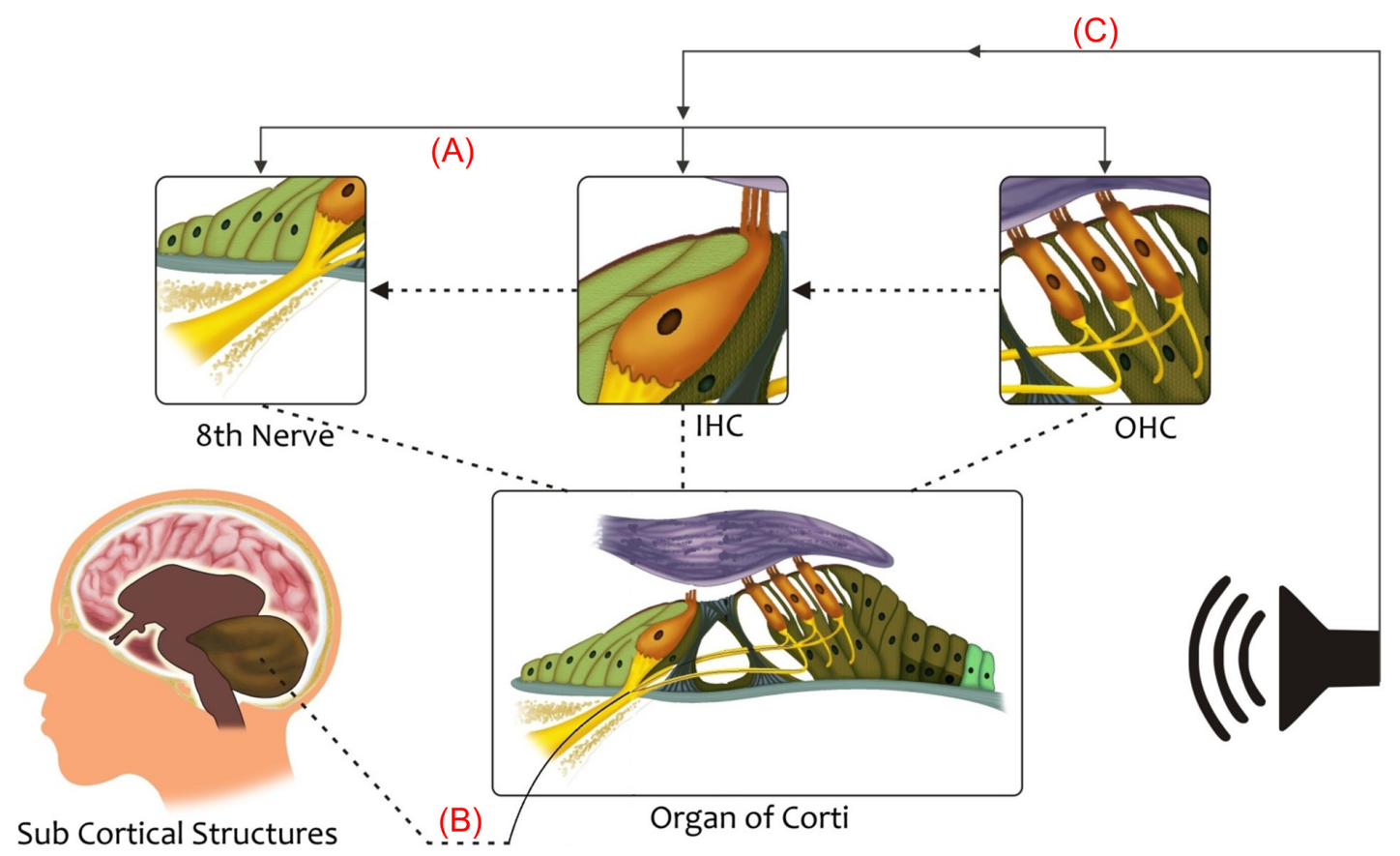

Figure 1. Hypothetical model to explain the co-existence of auditory dys-synchrony, hyperacusis and tinnitus based on their pathophysiology. Link 1 depicts the etiology leading to auditory dys-synchrony (which can be at the junction between the cochlear branch of VIII nerve and the inner hair cell that is the synapse, the inner hair cell or at the VIII nerve itself); link 2 represents hyperacusis (which arises due to compensation by central nervous system for a decrease in sensory input, by increasing the sensitivity of other centers involved in perception) and, link 3 represents tinnitus (due to both auditory dys-synchrony and hyperacusis, there is reduction in the central efferent suppression which leads to an increase in the gain of cochlear amplifier and results in tinnitus).

while the stereocilia of the inner hair cell make only a feeble connection. The basilar membrane is held below the inner hair cells while the outer hair cells are located near the center of the basilar membrane where vibrations will be greatest. This suggests that the resulting modulation of the ionic currents resulting from the movement of stereocilia is likely to be greater for outer hair cells than inner hair cells (23).

In the patient, there is co-existence of all the three conditions of auditory dys-synchrony, tinnitus and hyperacusis. Tinnitus, the perception of phantom sounds, and hyperacusis, the reduced tolerance for sounds, are often comorbid (12). Both hyperacusis and tinnitus arise from either sound-evoked or spontaneous hyperactivity in the auditory neural pathways. This reduction in peripheral neural activity occurs due to damage to sensory cells that reduces synaptic transmission to cochlear nerve terminals $(24,25)$. It is suggested that tinnitus and hyperacusis, result from a maladaptation of the central auditory system to peripheral dysfunction, while triggered by cochlear damage (26). Both tinnitus and hyperacusis could be explained in terms of a central gain model.

\section{Central gain mechanism}

The neural activity transmitted from the cochlea to the central auditory system is reduced in sensorineural hearing loss. Despite reduced output from the cochlea, there will be enhancement in neural activity in central auditory structures at suprathreshold levels (27). Tinnitus and hyperacusis, while triggered by cochlear damage, result from a maladaptation of the central auditory system to this peripheral dysfunction (26). As the feedback reaching from the central structures to the cochlea is not adequate, suppression will not take place and hence leads to over firing of the fibers. This is indicated through the outcomes from Oto-acoustic emissions which was robust for this patient.

Thus, central gain modulation is linked to loudness perception, which suggests that central gain enhancement may manifest as hypersensitivity to loudness, i.e., hyperacusis. To support this, the uncomfortable level of loudness for speech stimulus was found to be $>90$ and also moderate Hyperacusis in both ears as indicated in JHQ.

There was absence of middle ear reflexes and ABR in this patient which could be due to damage in the inner and outer hair cells. If only outer hair cells remain, emissions are seen, but without synchronous firing of the neural elements stemming from inner hair cells, which is seen in auditory dys-synchrony.

The compensatory increase in response to the loss of sensory input in the central auditory activity is referred to as central gain enhancement. According to the central gain mechanism, there will be recalibration of 
mean firing rate activity of the central auditory system to a new "set-point" due to lack of sensory input, thus generating an amplification of neural noise, which will be perceived as tinnitus. Notably, this neuronal recalibration can result in an enhancement of incoming sensory signals, which may increase the loudness intolerance and hyperacusis which often accompanies hearing loss (27). Hence, the Central Gain Model accounts for both tinnitus and hyperacusis.

Thus, the possible reason for the coexistence of these three conditions is explained as follows. As there is auditory dys-synchrony, the feedback to the OHC from the central gain mechanism will be affected and there will be a greater firing rate and hence over excitation of $\mathrm{OHC}$ even for less intense sounds. There will be compensation by the central nervous system for a decrease in sensory input, by increasing the sensitivity of other centers involved in perception (28). This may lead to intolerance to sound termed as hyperacusis ((B) in Figure 1).

Due to both auditory dys-synchrony and hyperacusis, there is reduction in central efferent suppression. This leads to an increase in the gain of cochlear amplifier and results in over activity related symptoms like tinnitus ((C) in Figure 1). A study demonstrates that tinnitus is accompanied by a change of the tonotopic map in the auditory cortex. A marked shift of the tinnitus frequency to the adjacent area was observed in the tonotopic location. There is also a positive association between subjective strength and the amount of shift of the tinnitus frequency in the auditory cortex. The reorganizational changes occur in the auditory cortex with both increase and decrease in input (26).

One of the rehabilitation options available for this condition is combination devices. Combination devices are one of the best treatments for tinnitus and hearing impairment as these devices mask the tinnitus and also amplify the sound to overcome the loss of hearing sensitivity. As this was not beneficial for the patient a cochlear implant was recommended. There are many studies which suggest the use of cochlear implants for people with auditory neuropathy spectrum disorder as these devices improved synchronous activity in the auditory nerve and help improve speech perception (29-31).

Link 1 represents the pathophysiology of auditory dys-synchrony which can be at the level of inner hair cell, junction of the inner hair cell and the cochlear branch of VIII nerve or at the VIII nerve itself. Link 2 represents the dys-function in the central gain mechanism which is responsible for hyperacusis. Link 3 explains the reduction in central gain mechanism due to both auditory dys-synchrony and hyperacusis which further leads to tinnitus. As there is auditory dyssynchrony, the feedback to the OHC from the central gain mechanism will be affected and there will be a greater firing rate and hence over excitation of $\mathrm{OHC}$ even for less intense sounds. The central nervous system will compensate for a decrease in sensory input, by increasing the sensitivity of other centers involved in perception. This may lead to intolerance to sound termed as hyperacusis. Due to both auditory dyssynchrony and hyperacusis, there is reduction in central efferent suppression, which leads to an increase in the gain of cochlear amplifier and results in over activity related symptoms like tinnitus.

In conclusion, the test results reported here of a single case study, imply that all three conditions can coexist as the way it is explained in the hypothetical model based on their pathophysiology. Tinnitus and hyperacusis result from a compensatory increase in gain in the central auditory system to compensate for a loss of cochlear sensory input.

\section{References}

1. National Council on the Aging (NCOA): The Impact of Untreated Hearing Loss in Older Americans. Conducted by the Seniors Research Group. Supported through a grant from the Hearing Industries Association. Preliminary report. 1998.

2. American Speech-Language-Hearing Association. Type, Degree, and Configuration of Hearing Loss. Audiology Information series. ASHA. 2011; 7976-16.

3. Rance G. Auditory neuropathy/ys-synchrony and its Perceptual Consequences. Trends in Amplif. 2005; 9:1-43.

4. Starr A, Picton TW, Sininger Y, Hood LJ, Berlin CI. Auditory neuropathy. Brain. 1996; 119:741-753.

5. Davis H, Hirsh S K. The audiometric utility of brain stem responses to low-frequency sounds. Audiology. 1976; 15:181-195.

6. Kumar UA, Jayaram MM. Prevalence and audiological characteristics in individuals with auditory neuropathy/ auditory dys-synchrony. Int J Audiol. 2006; 45:360-366.

7. Prabhu P, Avilala VKY, Manjula PP. Predisposing factors in individuals with late-onset auditory dys-synchrony. Asia Pac J Speech, Lang, Hear. 2012; 15:41-50.

8. McFadden D. Tinnitus: Facts, Theories and Treatments. Psychological Medicine. 1982; 13:699-699.

9. Han BI, Lee HW, Kim TY, Lim JS, Shin KS. Tinnitus: Characteristics, causes, mechanisms, and treatments. J Clin Neurol. 2009; 5:11-19.

10. Hoffman HJ, Reed GW. Epidemiology of tinnitus. In: Snow JB Jr, ed: Tinnitus: Theory and Management. Hamilton, Ontario: B.C. Decker, Inc. 2004; pp.16-41.

11. Møller AR. Tinnitus: presence and future. Prog Brain Res. 2007; 166:3-16.

12. Baguley DM. Hyperacusis, J R Soc Med. 2003; 96, 582585 .

13. Jastreboff MM, Jastreboff PJ. Hyperacusis, Audiology Online. http://www.audiologyonline.com/articles/ hyperacusis-1223 (accessed November 5, 2015)

14. Auo HJ, Park KH, Yeo SW, Chang KH, Choi HG, Choi BJ, Han MA, Park SN. Treatment response of modified tinnitus retraining therapy with medical therapy in the patients with tinnitus. Korean J OtorhinolaryngologyHead and Neck Surg. 2009; 52:648-654.

15. Tyler RS, Conrad-Armes D. The determination of tinnitus loudness considering the effects of recruitment. J Speech 
Hear Res. 1983; 26:59-72.

16. Berlin CI, Morlet T, Hood LJ. Auditory neuropathy/dyssynchrony: The diagnosis and management. Pediatr Clin North Am. 2003; 50:331-340.

17. Newman CW, Jacobson GP, Spitzer JB. Development of the tinnitus handicap inventory. Arch Otolaryngol Head Neck Surg. 1996; 122:143-148.

18. Amatuzzi MG, Northrop C, Liberman MC, Thornton A, Halpin C, Herrmann B, Pinto LE, Saenz A, Carranza A, Eavey RD. Selective inner hair cell loss in premature infants and cochlea pathological patterns from neonatal intensive care unit autopsies. Arch Otolaryngol Head Neck Surg. 2001; 127:629-636.

19. Berlin CI, Hood LJ, Cecola RP, Jackson DF, Szabo P. Does type I afferent neuron dysfunction reveal itself through lack of efferent suppression? Hear Res. 1993; 65:40-50.

20. Deltenre P, Mansbach AL, Bozet C, Christiaens F, Barthelemy P, Paulissen D, Renglet T. Auditory neuropathy with preserved cochlear micro-phonics and secondary loss of otoacoustic emissions. Audiology. 1999; 38:187-195.

21. Rance G, Beer DE, Cone-Wesson B, Shepherd RK, Dowell RC, King AM, Rickards FW, Clark GM. Clinical findings for a group of infants and young children with auditory neuropathy. Ear Hear. 1999; 20:238-252.

22. Wersinger E, Fuchs PA. Modualation of hair cell efferents. Hear Res. 2011; 279:1-12.

23. Ruggero MA, Rich NC. Systemic injection of furosemide alters the mechanical response to sound of the basilar membrane. In: The Mechanics and Biophysics of Hearing, Eds: P. Dallos et al, Springer, Berlin. 1990; 87:314-321.
24. Liberman MC, Dodds LW. Single-neuron labeling and chronic cochlear pathology. III. Stereocilia damage and alterations of threshold tuning curves. Hear Res. 1984; 16:55-74.

25. Liberman MC, Kiang NY. Acoustic trauma in cats: Cochlear pathology and auditory-nerve activity. Acta Otolaryngol Suppl. 1978; 358:1-63.

26. Mühlnickel W, Elbert T, Taub E, Flor H. Reorganization of auditory cortex in tinnitus. Proc Natl Acad Sci U S A. 1998; 95:10340-10343.

27. Auerbach BD, Rodrigues PV, Salvi RJ. Central gain control in tinnitus and hyperacusis. Front Neurol. 2014; 5:206.

28. Jastreboff PJ. Phantom auditory perception (tinnitus): mechanisms of generation and perception. Neurosci Res. $1990 ; 8: 221-54$.

29. Fabry LB. Identification and management of auditory neuropathy: case study. In: Seewald RC, editor. A sound foundation through early amplification: proceedings of an international conference. Chicago: Phonak; 2000. pp. 237245.

30. Trautwein PG, Sininger YS, Nelson R. Cochlear implantation of auditory neuropathy. J Am Acad Audiol. 2000; 11:309-315.

31. Shallop JK, Peterson A, Facer GW, Fabry LB, Driscoll CL. Cochlear implants in five cases of auditory neuropathy: Postoperative findings and progress. Laryngoscope. 2001; 111:555-562.

(Received November 6, 2015; Revised January 6, 2016; Accepted January 10, 2016) 